Article

\title{
MPC for LPV Systems Based on Parameter-Dependent Lyapunov Function with Perturbation on Control Input Strategy
}

\author{
Pornchai Bumroongsri and Soorathep Kheawhom* \\ Department of Chemical Engineering, Faculty of Engineering, Chulalongkorn University, Phayathai Rd., \\ Patumwan, Bangkok 10330, Thailand \\ E-mail: Soorathep.k@chula.ac.th*
}

\begin{abstract}
In this paper, the model predictive control (MPC) algorithm for linear parameter varying (LPV) systems is proposed. The proposed algorithm consists of two steps. The first step is derived by using parameter-dependent Lyapunov function and the second step is derived by using the perturbation on control input strategy. In order to achieve good control performance, the bounds on the rate of variation of the parameters are taken into account in the controller synthesis. An overall algorithm is proved to guarantee robust stability. The controller design is illustrated with two case studies of continuous stirredtank reactors. Comparisons with other MPC algorithms for LPV systems have been undertaken. The results show that the proposed algorithm can achieve better control performance.
\end{abstract}

Keywords: MPC, LPV, parameter-dependent Lyapunov function, perturbation on control input strategy, continuous stirred-tank reactor.

ENGINEERING JOURNAL Volume 16 Issue 2

Received 14 October 2011

Accepted 21 December 2011

Published 1 April 2012

Online at http://www.engj.org

DOI:10.4186/ej.2012.16.2.61 


\section{Introduction}

Model predictive control (MPC) is an effective control algorithm widely used in the chemical processes. At each sampling time, MPC uses an explicit process model to solve an open-loop optimization problem and implements only the first element of input sequence. Model predictive controllers based on linear models are typically used. This is because the on-line optimization problem can be formulated as the convex optimization problem by either linear programming or quadratic programming [1]. This is a good assumption for typical processes. However, most of the chemical processes are nonlinear. Thus, when the operating conditions undergo significant changes, the performance of linear MPC can deteriorate drastically. Moreover, the stability of the control system cannot be guaranteed.

In [1], min-max predictive control strategy was presented. The nonlinear system is approximated by the polytopic uncertain system. The goal is to design a state feedback control law which minimizes the upper bound on the worst-case performance cost. The optimization problem at each time step is formulated as the convex optimization problem involving linear matrix inequalities. The algorithm is proved to guarantee robust stability. However, the algorithm turns out to be very conservative. This is due to the fact that the nonlinear system is approximated by the polytopic uncertain system. Moreover, the scheduling parameter is not taken into account in the controller synthesis.

In order to reduce the conservativeness, the idea of controlling nonlinear systems by using linear parameter varying (LPV) systems has been widely investigated. At each sampling instant, the scheduling parameter is measured on-line. However, its future behaviour is considered to be uncertain and varying within the polytope. In [2], Quasi-min-max MPC algorithm for LPV systems was presented. The control input is computed by minimizing the upper bound on the quasi-worst-case performance cost. The algorithm is seen as an extension of the algorithm presented in [1] by keeping the first control input as a free decision variable. The algorithm is proved to guarantee robust stability. However, an invariant ellipsoid constructed to guarantee robust stability is derived by using a single Lyapunov function. Thus, the conservative result is still obtained.

A feedback min-max MPC algorithm for LPV systems subject to bounded rates of change of parameters was presented in [3]. The algorithm in [2], where open-loop MPC scheme is limited to one step control horizon, is extended to the general case of control horizon of arbitrary length $N$. The bounded parameter variations are assumed to be known. Moreover, they are exploited in the controller synthesis in order to improve control performance. However, the stability of the control system cannot be guaranteed. This is due to the fact that the constraint on terminal invariant set is not explicitly imposed. Moreover, input and output constraints satisfaction before switching horizon $N$ cannot be guaranteed as pointed out in [4].

The ability of on-line MPC is limited to relatively slow dynamics processes. In order to reduce on-line computational demand, a number of researchers have begun to study off-line MPC. In [5], an off-line formulation of MPC using linear matrix inequalities was presented. A sequence of explicit control laws corresponding to a sequence of invariant ellipsoids is constructed off-line by solving the optimization problem presented in [1]. At each sampling time, the smallest ellipsoid containing the measured state is determined and the real-time control law is calculated by linear interpolation between control laws of two adjacent invariant ellipsoids. The stability of the control system is proved to be guaranteed. However, the conservative result is obtained. This is due to the fact that the algorithm directly solves off-line the optimization problem presented in [1]. In [6], an ellipsoidal off-line MPC algorithm based on nominal performance cost was presented. The algorithm directly extends the algorithm presented in [5] by choosing the nominal performance cost to substitute the worst-case performance cost.

From the preceding review, we can see that robust stability is usually achieved with conservative result. In this paper, the closed-loop MPC strategy for LPV systems is developed. The proposed algorithm consists of two steps. The first step is derived by using parameter dependent Lyapunov function [7] and the second step is derived by using the perturbation on control input strategy $[3,8]$. The scheduling parameter and the bounded parameter variations are taken into account in the controller synthesis. Thus, the control performance is improved.

The paper is organized as follows. In section 2, the problem description is presented. In section 3 , the proposed MPC algorithm for LPV systems is presented. In section 4, we present two examples in chemical processes to illustrate our algorithm. Moreover, comparisons with other MPC algorithms have been undertaken. Finally, in section 5, we conclude the paper. 
Notation: For a matrix $A, A^{T}$ denotes its transpose, $A^{-1}$ denotes its inverse. $I$ denotes the identity matrix. For a vector $x, x(k / k)$ denotes the state measured at real time $k, x(k+i / k)$ denotes the state at prediction time $k+i$ predicted at real time $k$. The symbol $*$ denotes the corresponding transpose of the lower block part of symmetric matrices. $\chi_{K}^{k+i}(x(k))=\operatorname{Co}\left\{\bar{\Phi}_{K}\left(p^{k+i-1}\right) x(k)\right\}$ denotes the closed convex hull of all $i$ steps state trajectories from $x$ at time $k$ under the state feedback gain $K . \operatorname{vert}\left\{\chi_{K}^{k+i}(x(k))\right\}$ denotes all vertices of $\chi_{K}^{k+i}(x(k))$. The matrix inequality $A>B(A \geq B)$ means that $A$ and $B$ are square symmetric and $A-B$ is positive (semi-) definite.

\section{Problem description}

The model considered here is the following discrete-time LPV system:

$$
\begin{aligned}
& x(k+1)=A(p(k)) x(k)+B u(k) \\
& y(k)=C x(k)
\end{aligned}
$$

where $x(k)$ is the state of the plant, $u(k)$ is the control input and $y(k)$ is the plant output. We assume that the scheduling parameter $p(k)$ is measurable on-line at each sampling time $k$. Moreover, we assume that

$$
A(p(k)) \in \Omega, \Omega=\operatorname{Co}\left\{A_{1}, A_{2}, \ldots, A_{L}\right\}
$$

where $\Omega$ is the polytope, $C o$ denotes convex hull, $A_{j}$ are vertices of the convex hull and $L$ is the number of vertices of the convex hull. Any $A(p(k))$ within the polytope $\Omega$ is a linear combination of the vertices such that

$$
\begin{gathered}
A(p(k))=\sum_{j=1}^{L} p_{j}(k) A_{j} \\
\sum_{j=1}^{L} p_{j}(k)=1,0 \leq p_{j}(k) \leq 1
\end{gathered}
$$

Further, let

$$
\chi_{K}^{k+i}(x(k))=\operatorname{Co}\left\{\bar{\Phi}_{K}\left(p^{k+i-1}\right) x(k)\right\}
$$

denote the closed convex hull of all $i$-steps state trajectories from $x$ at time $k$ under the state-feedback gain $K$ where

$$
\Phi_{K}(p(k))=A(p(k))+B K
$$

$$
\bar{\Phi}_{K}\left(p^{k+i-1}\right)=\Phi_{K}(p(k)) \Phi_{K}(p(k+1)) \Phi_{K}(p(k+2)) \ldots \ldots \Phi_{K}(p(k+i-1))
$$

Following [8], the above sets can be computed according to

$$
\begin{gathered}
\chi_{K}^{k+i}(x(k))=\operatorname{Co}\left\{\Phi_{K}(p(k+i-1)) z, \forall z \in \operatorname{vert}\left\{\chi_{K}^{k+i-1}(x(k))\right\}, \forall p(k+i-1) \in \mathbf{P}^{\kappa+i-1}\right\} \\
\mathbf{P}^{\kappa+1}=\operatorname{vert}\left\{\operatorname{co}\left\{p(k)_{-}^{+} \Delta p\right\}\right\} \\
\mathbf{P}^{\kappa+2}=\operatorname{vert}\left\{\operatorname{co}\left\{p(k+1)_{-}^{+} \Delta p\right\}\right\} \\
\mathbf{P}^{\kappa+\mathbf{i}-1}=\operatorname{vert}\left\{\operatorname{co}\left\{p(k+i-2)_{-}^{+} \Delta p\right\}\right\}
\end{gathered}
$$

where $\Delta p$ is the bounded parameter variation and it is assumed to be known.

The aim of this research is to find a state feedback regulation

$$
u(k)=g(x(k))
$$

which stabilizes Eq. (1)-(3) and achieves the following performance cost

$$
\begin{aligned}
& \min _{u(k+i / k)} \max _{A(p(k+i)) \in \Omega, i \geq 0} J_{\infty}(k) \\
& J_{\infty}(k)=\sum_{i=0}^{\infty}\left[\begin{array}{l}
x(k+i / k) \\
u(k+i / k)
\end{array}\right]^{T}\left[\begin{array}{ll}
\Theta & 0 \\
0 & R
\end{array}\right]\left[\begin{array}{l}
x(k+i / k) \\
u(k+i / k)
\end{array}\right]
\end{aligned}
$$


where $\Theta>0$ and $R>0$ are symmetric weighting matrices, subject to constraints

$$
\begin{aligned}
& \left|u_{h}(k+i / k)\right| \leq u_{h, \max }, h=1,2,3 \ldots n_{u} \\
& \left|y_{r}(k+i / k)\right| \leq y_{r, \text { max }}, r=1,2,3 \ldots n_{y}
\end{aligned}
$$

In [1], the optimization problem Eq. (9) is formulated as the convex optimization problem involving linear matrix inequalities. The goal is to design the state feedback control law which minimizes the upper bound on the worst-case performance cost. The algorithm is derived by using a single Lyapunov function. Consequently, this approach turns out to be conservative. In order to reduce the conservativeness, MPC synthesis by using a parameter-dependent Lyapunov function is developed.

Lemma 2.1: Consider the LPV system (1) at each sampling timek, the state feedback control law which minimizes the upper bound $\gamma$ on the worst-case MPC objective $J_{\infty}(k)$ and asymptotically stabilizes the closed-loop system within an invariant ellipsoid $\varepsilon=\left\{x / x^{T} Q^{-1} x \leq 1, Q=\sum_{j=1}^{L} p_{j}(k) Q_{j}\right\} \quad$ is given by $u(k+i / k)=K(p(k+i)) x(k+i / k)$, $K(p(k+i))=\sum_{j=1}^{L} p_{j}(k+i) K_{j}, K_{j}=Y_{j} G_{j}^{-1}$ where $Y_{j}, G_{j}$ and $Q_{j}$ are the matrix variables obtained by solving the following problem:

$$
\begin{aligned}
& \min _{\gamma, Y_{j}, G_{j}, Q_{j}} \gamma \\
& \text { s.t. }\left[\begin{array}{cc}
1 & x(k / k)^{T} \\
x(k / k) & Q_{j}
\end{array}\right] \geq 0, \forall j=1,2 \ldots L \\
& {\left[\begin{array}{cccc}
G_{j}+G_{j}^{T}-Q_{j} & * & * & * \\
A_{j} G_{j}+B Y_{j} & Q_{l} & * & * \\
\Theta^{\frac{1}{2}} G_{j} & 0 & \gamma I & * \\
R^{\frac{1}{2}} Y_{j} & 0 & 0 & \gamma I
\end{array}\right] \geq 0, \forall j=1,2 \ldots L, \forall l=1,2 \ldots L} \\
& {\left[\begin{array}{cc}
X & * \\
Y_{j}^{T} & G_{j}+G_{j}^{T}-Q_{j}
\end{array}\right] \geq 0, \forall j=1,2 \ldots . L, X_{h h} \leq u_{h, \max }^{2}, h=1,2 \ldots n_{u}} \\
& {\left[\begin{array}{cc}
S & * \\
\left(A_{j} G_{j}+B Y_{j}\right)^{T} C^{T} & G_{j}+G_{j}^{T}-Q_{j}
\end{array}\right] \geq 0, \forall j=1,2 \ldots L, S_{r r} \leq y_{r, \max }^{2}, r=1,2 \ldots n_{y}}
\end{aligned}
$$

Proof. Proof details can be found in [7].

The existence of a symmetric matrix $X$ with $X_{h h} \leq u_{h, \max }^{2}, h=1,2, . . n_{u}$, such that $\left[\begin{array}{cc}X & * \\ Y^{T} & G_{j}+G_{j}^{T}-Q_{j}\end{array}\right] \geq 0, j=1,2, \ldots L$ guarantees that $\left|u_{h}(k+i / k)\right| \leq u_{h, \max }$ and the existence of a symmetric matrix $S$ with $S_{r r} \leq y_{r, \text { max }}^{2}, r=1,2, . . n_{y}$ such that $\left[\begin{array}{cc}S & * \\ \left(A_{j} G_{j}+B Y_{j}\right)^{T} C^{T} & G_{j}+G_{j}^{T}-Q_{j}\end{array}\right] \geq 0$, $j=1,2, \ldots L$ guarantees that $\left|y_{r}(k+i / k)\right| \leq y_{r, \max }$. For more details, the reader is referred to [9].

Another idea to reduce the conservativeness is to use the perturbation on control input strategy [3]. However, this strategy cannot guarantee robust stability. This is due to the fact that the terminal constraint is not explicitly imposed in the optimization problem. Thus, there's no constraint to guarantee that 
$x(k+N) \in \varepsilon$. Moreover, the input and output constraints satisfaction before switching horizon $N$ cannot be guaranteed [4]. In the following lemma, we will present the perturbation on control input strategy which can ensure robust stability and robust constraint satisfaction.

Lemma 2.2: Given the state feedback gain $K$ which asymptotically stabilizes the system (1) witbin an invariant ellipsoid $\varepsilon=\left\{x / x^{T} Q^{-1} x \leq 1\right\}$ and satisfies (10), (11), we can ensure that the system (1) is robustly stabilized and (10), (11) are satisfied by the sequence of control inputs

$$
u_{h}(k+i / k)=\left\{\begin{array}{c}
(K x(k+i / k)+c(k+i / k))_{h}, i=0,1, \ldots . N-1 \\
(K x(k+i / k))_{h}, i \geq N
\end{array}\right\}
$$

if there exists a sequence of free control inputs $\{c(k+i / k)\}_{i=0}^{N-1}$ satisfying the following inequalities:

$$
\begin{gathered}
z(k+N / k)^{T} Q^{-1} z(k+N / k) \leq 1, \forall z(k+N / k) \in \operatorname{vert}\left\{\chi_{K}^{k+N}(x(k))\right\} \\
\left|(K z(k+i / k)+c(k+i / k))_{h}\right| \leq u_{h, \text { max }}, \forall i \in\{0,1, \ldots N-1\}, \\
\forall h \in\left\{1,2 \ldots N_{u}\right\}, \forall z(k+i / k) \in \operatorname{vert}\left\{\chi_{K}^{k+i}(x(k))\right\} \\
\left|(C z(k+i / k))_{r}\right| \leq y_{r, \max }, \forall i \in\{0,1, \ldots . N-1\}, \\
\forall r \in\left\{1,2 \ldots N_{y}\right\}, \forall z(k+i / k) \in \operatorname{vert}\left\{\chi_{K}^{k+i}(x(k))\right\}
\end{gathered}
$$

Proof. The satisfaction of (17) ensures that $x(k+N / k) \in \varepsilon, \forall x(k+N / k) \in \chi_{K}^{k+N}(x(k))$. If $x(k+N / k) \in \varepsilon$, the state feedback gain $K$ is able to steer the state from $x(k+N / k)$ to the origin. Thus, the closed-loop system is robustly stabilized.

The satisfaction of (18) ensures that $\left|(K x(k+i / k)+c(k+i / k))_{h}\right| \leq u_{h, \max }, \forall x(k+i / k) \in \chi_{K}^{k+i}(x(k))$. Thus, (10) must be satisfied. The satisfaction of (19) ensures that $\left|(C x(k+i / k))_{r}\right| \leq y_{r, \max }$, $\forall x(k+i / k) \in \chi_{K}^{k+i}(x(k))$. Thus, (11) must be satisfied.

\section{The proposed MPC algorithm for LPV systems}

In this section, the MPC algorithm for LPV systems is proposed. Lemmas 1 and 2 will be used in the controller synthesis. The proposed algorithm consists of two steps. In the first step, the state feedback control law is calculated by using parameter-dependent Lyapunov function. The state feedback control law provided by the first step is designed to robustly stabilize the closed-loop system. In the second step, the state feedback control law calculated from step 1 is perturbed by using a sequence of free control inputs $\{c(k+i / k)\}_{i=0}^{N-1}$. The control performance is improved by minimizing an additional performance cost over a perturbation horizon $N$. The scheduling parameter and the bounds on the rate of variation of the parameters are taken into account in the problem formulation.

\section{Algorithm 3.1}

\section{Step 1:}

At any sampling time $k$, measure $x(k)$ and find $\gamma, K_{j}=Y_{j} G_{j}^{-1}$ and $Q_{j}$ by solving the optimization problem in Lemma 2.1 [7].

\section{Step 2:}

Given $\gamma, K_{j}=Y_{j} G_{j}^{-1}$ and $Q_{j}$ from step 1 , a sequence of free control inputs $\{c(k+i / k)\}_{i=0}^{N-1}$ is obtained by solving the following problem 


$$
\begin{aligned}
& \min _{J_{i}>0, C_{\text {opt }}} \sum_{i=0}^{N} J_{i} \\
& \text { s.t. }\left[\begin{array}{cc}
J_{N} & * \\
z(k+N / k) & \gamma^{-1} Q_{j}
\end{array}\right] \geq 0, \forall j=1,2 \ldots L, \forall z(k+N / k) \in \operatorname{vert}\left\{\chi_{K}^{k+N}(x(k))\right\} \\
& J_{N} \leq \gamma \\
& \left|\left(K_{j} z(k+i / k)+c(k+i / k)\right)_{h}\right| \leq u_{h, \max }, \forall i \in\{0,1 \ldots N-1\}, \\
& \forall h \in\left\{1,2 \ldots N_{u}\right\}, \forall z(k+i / k) \in \operatorname{vert}\left\{\chi_{K}^{k+i}(x(k))\right\} \\
& \left|(C z(k+i / k))_{r}\right| \leq y_{r, \max }, \forall i \in\{0,1, \ldots . . N-1\}, \\
& \forall r \in\left\{1,2 \ldots N_{y}\right\}, \forall z(k+i / k) \in \operatorname{vert}\left\{\chi_{K}^{k+i}(x(k))\right\} \\
& {\left[\begin{array}{ccc}
I & * & * \\
\Theta^{\frac{1}{2}} z(k+i / k) & J_{i} I_{n_{x}} & * \\
R^{\frac{1}{2}} c(k+i / k) & 0 & J_{i} I_{n_{u}}
\end{array}\right] \geq 0} \\
& \forall i \in\{0,1, \ldots . N-1\}, \forall z(k+i / k) \in \operatorname{vert}\left\{\chi_{K}^{k+i}(x(k))\right\}
\end{aligned}
$$

Feed the plant by $u(k)=K(p(k)) x(k)+c(k), K(p(k))=\sum_{j=1}^{L} p_{j}(k) K_{j}$.

The stability of the control system is proved to be guaranteed in Theorem 3.1.

Theorem 3.1: The control law provided by the algorithm 3.1 assures robust stability to the closed-loop system.

Proof. We prove this theorem in two steps. In step a, we prove that the control law $u(k+i / k)=K(p(k+i)) x(k+i / k), K(p(k+i))=\sum_{j=1}^{L} p_{j}(k+i) K_{j}, K_{j}=Y_{j} G_{j}^{-1}, i \geq 0 \quad$ provided $\quad$ by $\quad$ step 1 asymptotically stabilizes the closed-loop system. In step b, we prove that by using the perturbations of $\{c(k+i / k)\}_{i=0}^{N-1}$ to improve control performance, the control law

$u(k+i / k)=\left\{\begin{array}{c}K(p(k+i)) x(k+i / k)+c(k+i / k), i=0,1, \ldots N-1 \\ K(p(k+i)) x(k+i / k), i \geq N\end{array}\right\}$

provided by step 2 asymptotically stabilizes the closed-loop system.

Step a) The proof is based on the same rationale used for proving in Lemma 2.1. Thus, the control law $u(k+i / k)=K(p(k+i)) x(k+i / k)$ provided by step 1 asymptotically stabilizes the closed-loop system.

Step b) By applying Schur complement to (20), with (21), we obtain $z(k+N / k)^{T} \gamma Q^{-1} z(k+N / k) \leq J_{N} \leq \gamma$ where $Q=\sum_{j=1}^{L} p_{j}(k) Q_{j}$. This is equivalent to $z(k+N / k)^{T} Q^{-1} z(k+N / k) \leq 1, \forall z(k+N / k) \in \operatorname{vert}\left\{\chi_{K}^{k+N}(x(k))\right\}$. Thus, the state $x(k+N / k)$ is restricted to lie in an invariant ellipsoid $\varepsilon=\left\{x / x^{T} Q^{-1} x \leq 1\right\}$ and the control law $u(k+i / k)=K(p(k+i)) x(k+i / k), i \geq N$ is able to steer the state from $x(k+N / k)$ to the origin. 


\section{Examples}

In this section, we present two examples that illustrate the implementation of the proposed MPC algorithm. For both examples, the numerical simulations have been performed in Intel Core i-5 (2.4 GHz), 2 GB RAM, using SeDuMi [10] and YALMIP [11] within Matlab R2008a environment.

Example 4.1: Consider the following nonlinear model for CSTR where the consecutive reaction $A \longrightarrow B \longrightarrow C$ takes place [12]

$$
\begin{aligned}
& x_{1}=-x_{1}-D a_{1} x_{1}+u \\
& \dot{x}_{2}=D a_{1} x_{1}-x_{2}-D a_{2} x_{2}^{2}
\end{aligned}
$$

where $x_{1}$ denotes the dimensionless concentration of $A, x_{2}$ denotes the dimensionless concentration of $B$, The control variable u corresponds to the inlet concentration of $A$. The operating parameters are shown in Table 1 . It is assumed that $A \longrightarrow B$ is a first order chemical reaction whereas $B \longrightarrow C$ is a second order chemical reaction.

Table 1. The operating parameters of nonlinear CSTR in example 4.1.

\begin{tabular}{lr}
\hline Parameter & Value \\
\hline$D a_{1}$ & 1 \\
$D a_{2}$ & 2 \\
\hline
\end{tabular}

By defining the deviation variables $\bar{x}_{1}=x_{1}-x_{1, e q}, \bar{x}_{2}=x_{2}-x_{2, e q}, \bar{u}=u-u_{e q}$ where the subscript $e q$ is used to denote the corresponding variable at equilibrium condition, we have that all the solutions of (26) are also the solutions of the following differential inclusion

$$
\left[\begin{array}{c}
\dot{\bar{x}}_{1} \\
\dot{\bar{x}}_{2}
\end{array}\right] \in\left(\sum_{j=1}^{2} p_{j} \Phi_{j}\right)\left[\begin{array}{l}
\bar{x}_{1} \\
\bar{x}_{2}
\end{array}\right]+\left[\begin{array}{l}
1 \\
0
\end{array}\right] \bar{u}
$$

where $\Phi_{j}$ is given by

$$
\Phi_{1}=\left[\begin{array}{cc}
-1-D a_{1} & 0 \\
D a_{1} & -1-D a_{2} x_{2, \min }
\end{array}\right], \Phi_{2}=\left[\begin{array}{cc}
-1-D a_{1} & 0 \\
D a_{1} & -1-D a_{2} x_{2, \max }
\end{array}\right]
$$

Consider $x_{2, \min } \leq x_{2} \leq x_{2, \max }$, the parameter $p_{1}$ is given by $p_{1}=\frac{x_{2, \max }-x_{2}}{x_{2, \max }-x_{2, \min }}$ and the parameter $p_{2}$ is given by $p_{2}=\frac{x_{2}-x_{2, \min }}{x_{2, \max }-x_{2, \min }}$. In this example, we have two controlled variables $\bar{x}_{1}, \bar{x}_{2}$ and one manipulated variable $\bar{u}$. The objective is to regulate $\bar{x}_{1}$ and $\bar{x}_{2}$ from 0.05 and 0.1 respectively to the origin by manipulating $\bar{u}$. The input and output constraints are given as follows:

$$
\begin{aligned}
& \left|\bar{x}_{1}\right| \leq 2 \\
& \left|\overline{\mathrm{x}}_{2}\right| \leq 2 \\
& |\bar{u}| \leq 2.5
\end{aligned}
$$


The discrete-time model is obtained by discretizing (27) using Euler discretization method with sampling time of $0.1 \mathrm{~min}$ and it is omitted here for brevity. Here $J_{\infty}(k)$ is given by (9) with $\Theta=10 I$ and $R=0.01$. It is assumed that $\left|p_{j}(k+1)-p_{j}(k)\right| \leq 0.1$.

The proposed MPC algorithm will be compared with the MPC algorithms of Kothare et al. [1], Lu et al. [2] and Wada et al. [7]. Figure 1 shows the closed-loop responses of the system. It can be observed from the figure that the proposed algorithm can achieve less conservative result as compared with other MPC algorithms.
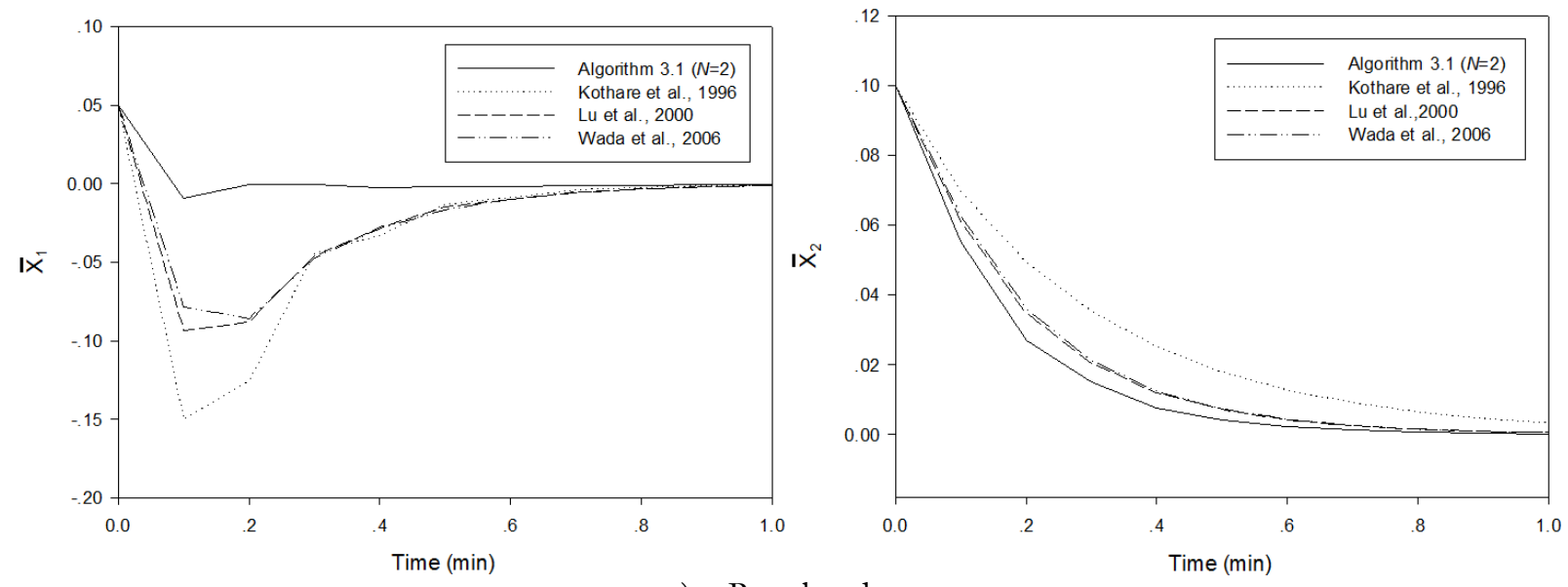

a) Regulated output

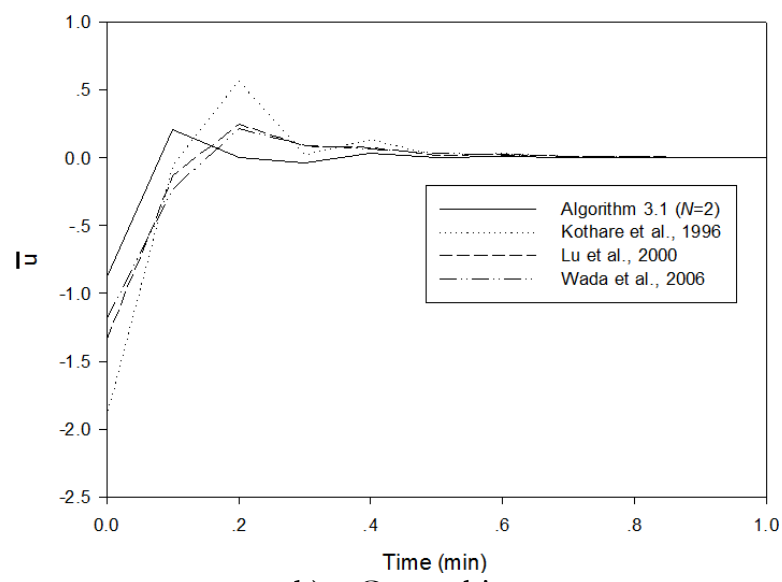

b) Control input

Fig. 1. The closed-loop responses of nonlinear CSTR in example 4.1.

In [1], the state feedback control law is designed by minimizing the upper bound on the worst-case performance cost. The quadratic function of the state is forced to decrease at each prediction time by the amount of the worst-case performance cost. However, the algorithm turns out to be very conservative. This is due to the fact that the nonlinear system is approximated by the polytopic uncertain system. Moreover, the scheduling parameter is considered to be uncertain and it is not taken into account in the controller synthesis.

In [2], the control input is computed by minimizing the upper bound on the quasi-worst-case performance cost. The algorithm is seen as an extension of the algorithm presented in [1] by keeping the first control input as a free decision variable. The scheduling parameter is measured on-line and it is incorporated into the problem formulation. However, an invariant ellipsoid constructed to guarantee robust stability is derived by using a single Lyapunov function. Thus, the conservative result is obtained.

In [7], the MPC algorithm is derived by using parameter dependent Lyapunov function. The scheduling parameter is measured on-line and it is taken into account in the controller design. However, the control input only depends on the state evolution $(u(k)=K(p(k)) x(k))$. Thus, the conservative result is still obtained. 
Figure 2 shows the cumulative cost $\sum_{i=0}^{t}\left[\bar{x}_{1}(i) \bar{x}_{2}(i)\right] \Theta\left[\bar{x}_{1}(i) \bar{x}_{2}(i)\right]^{T}+\bar{u}(i) R \bar{u}(i)$. It can be observed that the proposed algorithm gives the lowest cost value as compared to MPC algorithms of Kothare et al. [1], Lu et al. [2] and Wada et al. [7].

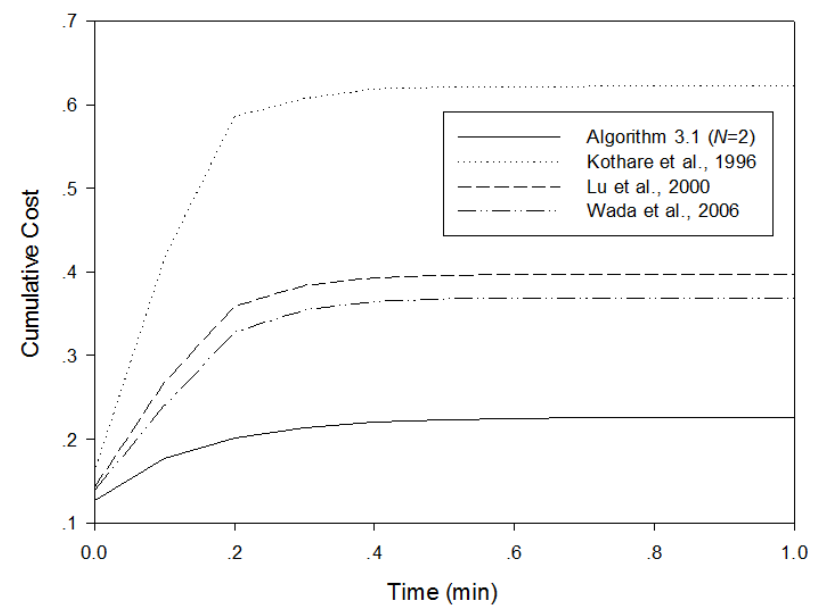

Fig. 2. The cumulative cost $\sum_{i=0}^{t}\left[\bar{x}_{1}(i) \bar{x}_{2}(i)\right] \Theta\left[\bar{x}_{1}(i) \bar{x}_{2}(i)\right]^{T}+\bar{u}(i) R \bar{u}(i)$ in example 4.1.

Though the proposed algorithm can achieve less conservative result as compared with other MPC algorithms, it requires higher on-line computational time as shown in Table 2.

Table 2. The on-line computational time in example 4.1.

\begin{tabular}{lr}
\hline Algorithm & CPU time per step \\
\hline Algorithm 3.1 & Step 1 $=0.196 \mathrm{~s}$ \\
$N=2$ & Step 2 $=0.153 \mathrm{~s}$ \\
\hline Kothare et al. & $0.142 \mathrm{~s}$ \\
Lu et al. & $0.157 \mathrm{~s}$ \\
Wada et al. & $0.196 \mathrm{~s}$ \\
\hline
\end{tabular}

Example 4.2: Consider the following nonlinear model for CSTR where the exothermic reaction $A \longrightarrow B$ takes place [13].

$$
\begin{aligned}
& \dot{C}_{A}=\frac{q}{V}\left(C_{A F}-C_{A}\right)-k_{o} e^{\left(-\frac{E a}{R T}\right)} C_{A} \\
& \dot{T}=\frac{q}{V}\left(T_{f}-T\right)+\frac{-\Delta H}{\rho C_{p}} k_{o} e^{\left(-\frac{E a}{R T}\right)} C_{A}+\frac{U A}{V \rho C_{p}}\left(T_{c}-T\right)
\end{aligned}
$$

where $C_{A}$ denotes the concentration of $A$ in the reactor, $T$ denotes the reactor temperature and $T_{c}$ denotes the temperature of coolant stream. The operating parameters are shown in Table 3. 
Table 3. The operating parameters of nonlinear CSTR in example 4.2.

\begin{tabular}{lcr}
\hline Parameter & Value & Unit \\
\hline$q$ & 100 & $1 / \mathrm{min}$ \\
$T_{f}$ & 350 & $\mathrm{~K}$ \\
$C_{A F}$ & 1 & $\mathrm{~mol} / 1$ \\
$V$ & 100 & 1 \\
$\rho$ & 1,000 & $\mathrm{~g} / \mathrm{l}$ \\
$C_{p}$ & 0.239 & $\mathrm{~J} / \mathrm{g} \mathrm{K}$ \\
$\Delta H$ & $-5 \times 10^{4}$ & $\mathrm{~J} / \mathrm{mol}$ \\
$E_{a} / R$ & 8,750 & $\mathrm{~K}$ \\
$k_{o}$ & $7.2 \times 10^{10}$ & $\mathrm{~min}^{-1}$ \\
$U A$ & $5 \times 10^{4}$ & $\mathrm{~J} / \mathrm{min} \mathrm{K}$ \\
\hline
\end{tabular}

By defining the deviation variables $\bar{C}_{A}=C_{A}-C_{A, e q}, \bar{T}=T-T_{e q}, \bar{T}_{c}=T_{c}-T_{c, e q}$ where the subscript $e q$ is used to denote the corresponding variable at equilibrium condition, we have that all the solutions of (30) are also the solutions of the following differential inclusion

$$
\left[\begin{array}{c}
\dot{\bar{C}}_{A} \\
\dot{\bar{T}}
\end{array}\right] \in\left(\sum_{j=1}^{2} p_{j} \Phi_{j}\right)\left[\begin{array}{c}
\bar{C}_{A} \\
\bar{T}
\end{array}\right]+\left[\begin{array}{c}
0 \\
\frac{U A}{V \rho C_{p}}
\end{array}\right] \bar{T}_{\mathrm{c}}
$$

where $\Phi_{j}$ is given by

$$
\Phi_{1}=\left[\begin{array}{cc}
-k_{o} e^{\left(-\frac{E_{a}}{R T_{\min }}\right)}-\frac{q}{V} & 0 \\
-\frac{\Delta H}{\rho C_{p}} k_{o} e^{\left(-\frac{E a}{R T_{\min }}\right)} & -\frac{q}{V}-\frac{U A}{V \rho C_{p}}
\end{array}\right], \Phi_{2}=\left[\begin{array}{cc}
-k_{o} e^{\left(-\frac{E_{a}}{R T_{\max }}\right)}-\frac{q}{V} & 0 \\
-\frac{\Delta H}{\rho C_{p}} k_{o} e^{\left(-\frac{E a}{R T_{\max }}\right)} & -\frac{q}{V}-\frac{U A}{V \rho C_{p}}
\end{array}\right]
$$

Consider $T_{\min } \leq T \leq T_{\max }$, the parameter $p_{1}$ is given by $p_{1}=\frac{e^{\left(-\frac{E_{a}}{R T_{\max }}\right)}-e^{\left(-\frac{E_{a}}{R T}\right)}}{e^{\left(-\frac{E_{a}}{R T_{\max }}\right)}-e^{\left(-\frac{E_{a}}{R T_{\min }}\right)}}$ and the parameter $p_{2}$ is given by $p_{2}=\frac{e^{\left(-\frac{E_{a}}{R T}\right)}-e^{\left(-\frac{E_{a}}{R T_{\min }}\right)}}{e^{\left(-\frac{E_{a}}{R T_{\max }}\right)}-e^{\left(-\frac{E_{a}}{R T_{\min }}\right)}}$. In this example, we have two controlled variables $\bar{C}_{A}, \bar{T}$ and one manipulated variable $\bar{T}_{c}$.The objective is to regulate $\bar{C}_{A}$ and $\bar{T}$ from 0.2 and 0.5 respectively to the origin by manipulating $\bar{T}_{c}$. The input and output constraints are given as follows:

$$
\begin{aligned}
& \left|\bar{C}_{A}\right| \leq 0.5 \mathrm{~mol} / \mathrm{l} \\
& |\overline{\mathrm{T}}| \leq 50 \mathrm{~K} \\
& \left|\overline{T_{c}}\right| \leq 50 \mathrm{~K}
\end{aligned}
$$

The discrete-time model is obtained by discretizing (31) using Euler discretization method with sampling time of $0.01 \mathrm{~min}$ and it is omitted here for brevity. Here $J_{\infty}(k)$ is given by (9) with $\Theta=I$ and $R=0.01$. It is assumed that $\left|p_{j}(k+1)-p_{j}(k)\right| \leq 0.1$.

Figure 3 shows the closed-loop responses of the system. It is seen from the figure that all algorithms behave almost identically in regulating the concentration of $\mathrm{A}\left(\overline{\mathrm{C}}_{A}\right)$. However, the proposed algorithm outperforms other algorithms in regulating the reactor temperature $\bar{T}$. 

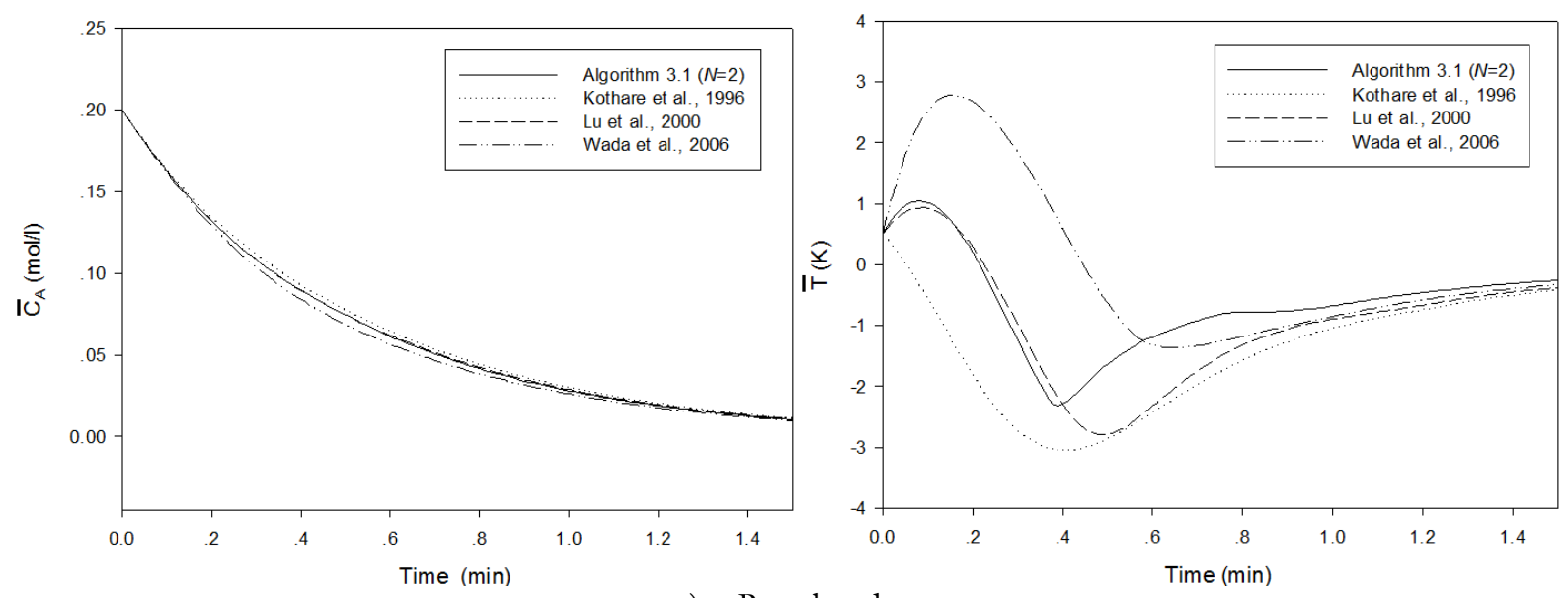

a) Regulated output

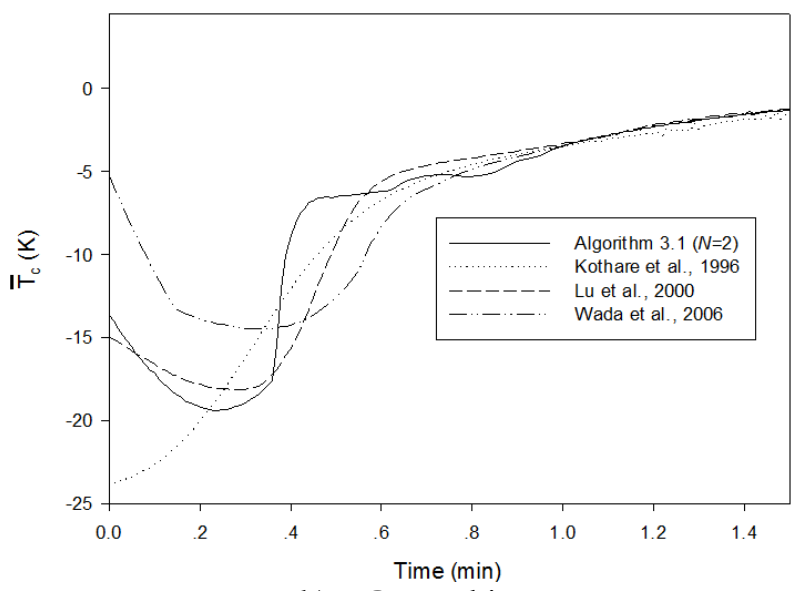

b) Control input

Fig. 3. The closed-loop responses of nonlinear CSTR in example 4.2.

Figure 4 shows the cumulative cost $\sum_{i=0}^{t}\left[\bar{C}_{A}(i) \bar{T}(i)\right] \Theta\left[\bar{C}_{A}(i) \bar{T}(i)\right]^{T}+\bar{T}_{c}(i) R \bar{T}_{c}(i)$. It can be observed from the figure that the proposed algorithm can achieve better control performance as compared to MPC algorithms of Kothare et al. [1], Lu et al. [2] and Wada et al. [7].

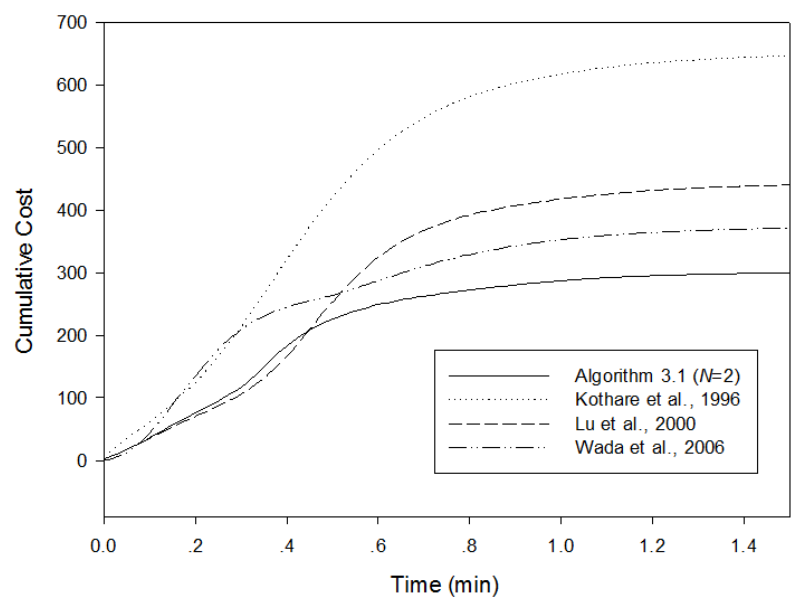

Fig. 4. The cumulative cost $\sum_{i=0}^{t}\left[\bar{C}_{A}(i) \bar{T}(i)\right] \Theta\left[\bar{C}_{A}(i) \bar{T}(i)\right]^{T}+\bar{T}_{c}(i) R \bar{T}_{c}(i)$ in example 4.2. 


\section{Conclusions}

In this paper, the synthesis approach to MPC for LPV systems using linear matrix inequalities is developed. The proposed algorithm consists of two steps. The first step is derived by using parameter dependent Lyapunov function and the second step is derived by using the perturbation on control input strategy. The bounds on the rate of variation of the parameters are taken into account in the controller synthesis in order to improve control performance. The controller design is illustrated with two examples in chemical processes. Comparisons with other MPC algorithms have been undertaken. The results show that the proposed algorithm can achieve better control performance.

\section{Acknowledgement}

The authors gratefully acknowledge the financial support provided by the Higher Education Research Promotion and National Research University Project of Thailand, Office of the Higher Education Commission (EN636A).

\section{References}

[1] M.V. Kothare, V. Balakrishnan, and M. Morari, "Robust constrained model predictive control using linear matrix inequalities," Automatica, vol. 32, pp. 1361-1379, 1996.

[2] Y. Lu and Y. Arkun, "Quasi-min-max MPC algorithms for LPV systems," Automatica, vol. 36, pp. 527$540,2000$.

[3] A. Casavola, D. Famularo, and G. Franze, "A feedback min-max MPC algorithm for LPV systems subject to bounded rates of change of parameters," IEEE T. Automat. Contr., vol. 47, pp. 1147-1152, 2002.

[4] B. Ding and B. Huang, "Comments on a feedback min-max MPC algorithm for LPV systems subject to bounded rates of change of parameters," IEEE T. Automat. Contr., vol. 52, pp. 970, 2007.

[5] Z. Wan and M.V. Kothare, "An efficient off-line formulation of robust model predictive control using linear matrix inequalities," Automatica, vol. 39, pp. 837-846, 2003.

[6] B. Ding, Y. Xi, M. T. Cychowski, and T. O. Mahony, "Improving off-line approach to robust MPC based-on nominal performance cost," Automatica, vol. 43, pp. 158-163, 2007.

[7] N. Wada, K. Saito, and M. Saeki, "Model predictive control for linear parameter varying systems using parameter dependent lyapunov function," IEEE T. Circuits Syst., vol.53, pp. 1446-1450, 2006.

[8] A. Casavola, D. Famularo, G. Franze, "Predictive control of constrained nonlinear systems via LPV linear embeddings," Int. J. Robust Nonlin., vol. 13, pp. 281-294, 2003.

[9] F. A. Cuzzola, J. C. Geromel, and M. Morari, "An improved approach for constrained robust model predictive control," Automatica, vol. 38, pp. 1183-1189, 2002.

[10] J. F. Sturm, "Using Sedumi 1.02, a MATLAB toolbox for optimization over symmetric cones," Optim. Method Softw., vol. 11, pp. 625-653, 1999.

[11] J. Löfberg, "YALMIP : A toolbox for modelling and optimization in MATLAB," in Proceedings of the 2004 IEEE international symposium on computer aided control systems design, Taipei, Taiwan, 2004, pp. 284289.

[12] J. P. García-Sandoval, V. González-Álvarez, B. Castillo-Toledo, and C. Pelayo-Ortiz "Robust discrete control of nonlinear processes: Application to chemical reactors," Comput. Chem. Eng., vol. 32, pp. 3246-3253, 2008.

[13] L. Magni, G.D. Nicolao, L. Magnani, and R. Scattolini "A stabilizing model-based predictive control algorithm for nonlinear system," Automatica, vol. 37, pp. 1351-1362, 2001. 